\title{
Asymptotic Behavior of Global Entropy Solutions for Nonstrictly Hyperbolic Systems with Linear Damping
}

\author{
Richard Alexander De la Cruz Guerrero, ${ }^{1}$ \\ Juan Carlos Juajibioy Otero, ${ }^{2}$ and Leonardo Rendon ${ }^{2}$ \\ ${ }^{1}$ School of Mathematics and Statistics, Pedagogical and Technological University of Colombia, Tunja, Colombia \\ ${ }^{2}$ Department of Mathematics, National University of Colombia, Bogotá, Colombia
}

Correspondence should be addressed to Juan Carlos Juajibioy Otero; jcjuajibioyo@unal.edu.co

Received 2 September 2014; Accepted 2 November 2014; Published 18 November 2014

Academic Editor: Salim Messaoudi

Copyright (C) 2014 Richard A. De la cruz Guerrero et al. This is an open access article distributed under the Creative Commons Attribution License, which permits unrestricted use, distribution, and reproduction in any medium, provided the original work is properly cited.

We investigate the large time behavior of the global weak entropy solutions to the symmetric Keyfitz-Kranzer system with linear damping. It is proved that as $t \rightarrow \infty$ the entropy solutions tend to zero in the $L^{p}$ norm.

\section{Introduction}

In this paper, we consider the Cauchy problem to the symmetric system of Keyfitz-Kranzer type with linear damping

$$
\begin{aligned}
& u_{t}+(u \phi(r))_{x}+a u=0, \\
& v_{t}+(v \phi(r))_{x}+b v=0,
\end{aligned}
$$

with initial data

$$
u(x, 0)=u_{0}(x), \quad v(x, 0)=u_{0}(x) .
$$

This system models the propagation models of propagation of forward longitudinal and transverse waves of elastic string which moves in a plane; see $[1,2]$. General source term for the system (1) was considered in [3]. The damping term in the system (1) represents external forces proportional to velocity, and this term can produce loss of total energy of system. Consider the scalar case; for example,

$$
u_{t}+a u_{x}+b u=0, \quad u(x, 0)=u_{0}(x) .
$$

From the integral representation of (3), it is easy to find the following solution:

$$
u(x, t)=u_{0}(x-a t) e^{-b t} .
$$

In this case, the solution of (3) tends to zero when $t \rightarrow \infty$. In Figure 1, we show the graph of solution for the advection equation with initial data

$$
u(x, 0)= \begin{cases}\left(1-x^{2}\right), & \text { if } x \in(-1,1) \\ 0, & \text { otherwise }\end{cases}
$$

For more general case, the behavior of solutions and its computation can be more complicated; for example, we consider Burger's equation with a particular initial data and linear damping; this equation models the component of the velocity in one-dimensional flow

$$
u_{t}+\left(\frac{1}{2} u^{2}\right)_{x}+u=0
$$

with intial data

$$
u(x, 0)= \begin{cases}A\left(1-x^{2}\right), & \text { if } x \in(-1,1), \\ 0, & \text { otherwise, }\end{cases}
$$

where $A$ is a constant. By an application to the characteristics method, we have that solutions emanating from $x_{0}$ are given by

$$
\begin{gathered}
X\left(t, x_{0}\right)=x_{0}+A\left(1-x_{0}^{2}\right)\left(1-e^{-t}\right), \\
u(X, t)=u_{0}(x) e^{-t} .
\end{gathered}
$$




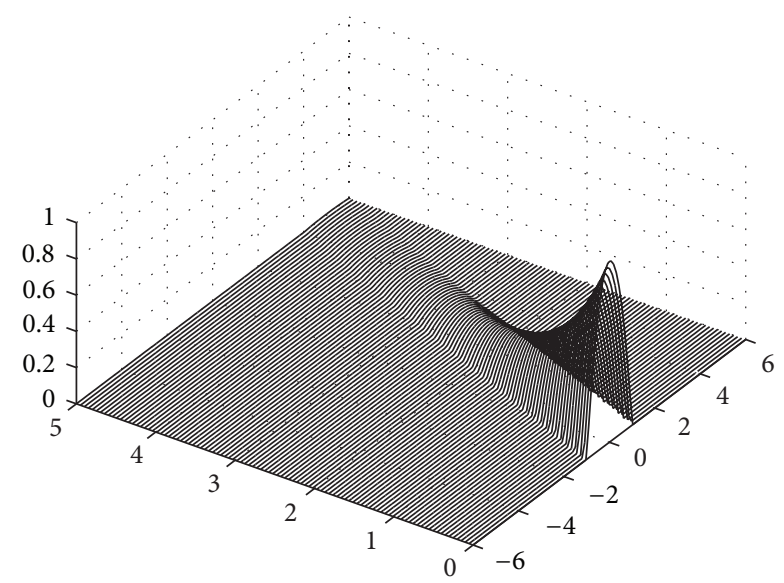

FIGURE 1: Graph of solution $u_{t}+u_{x}+u=0, A=1$.

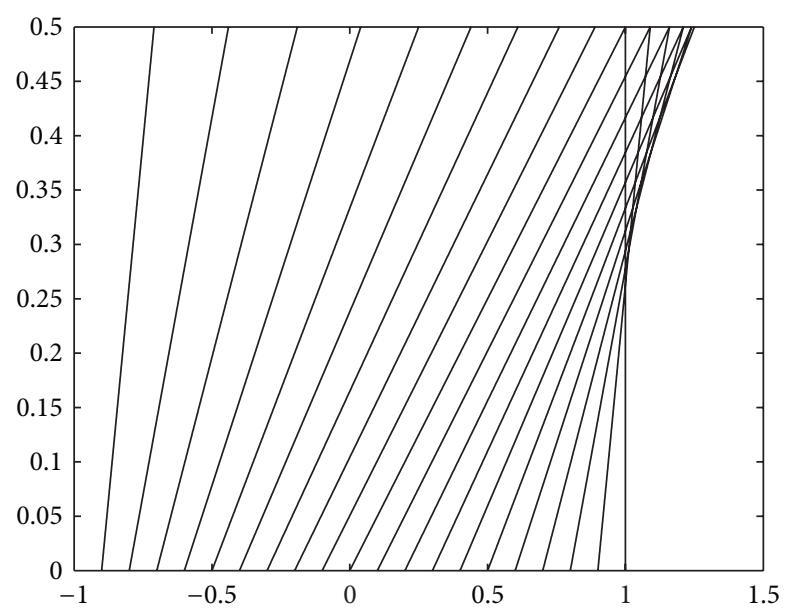

FIgURE 2: Characteristics for $u=0, A=1$.

In general, in systems with source term, the characteristics are nonlinear functions and they could have asymptotic behavior; for example, the characteristics solutions for (6), (7) are asymptotic to the lines

$$
\lim _{t \rightarrow \infty} X\left(t, x_{0}\right)=x_{0}+A\left(1-x_{0}^{2}\right) .
$$

It is easy to see that the shock occurs when $x_{0}+A\left(1-x_{0}\right)^{2}>1$. In Figures 2, 3, and 4, we show the characteristics solutions for several values of $A$.

We are looking for conditions under which the terms $a, b$ have a dissipative effect in the solutions of (1).

Let $r(x, t)=\sqrt{u(x, t)^{2}+v(x, t)^{2}}$, and we are going to show the following main theorem.

Theorem 1. If the initial data $\left(u_{0}(x), v_{0}(x)\right) \in L^{\infty}(\mathbb{R}) \cap$ $L^{2}(\mathbb{R})$, then the Cauchy problems (1) and (2) have a weak entropy solutions satisfying

$$
\|u\|_{L^{\infty}(\Omega)}+\|v\|_{L^{\infty}(\Omega)}<M
$$

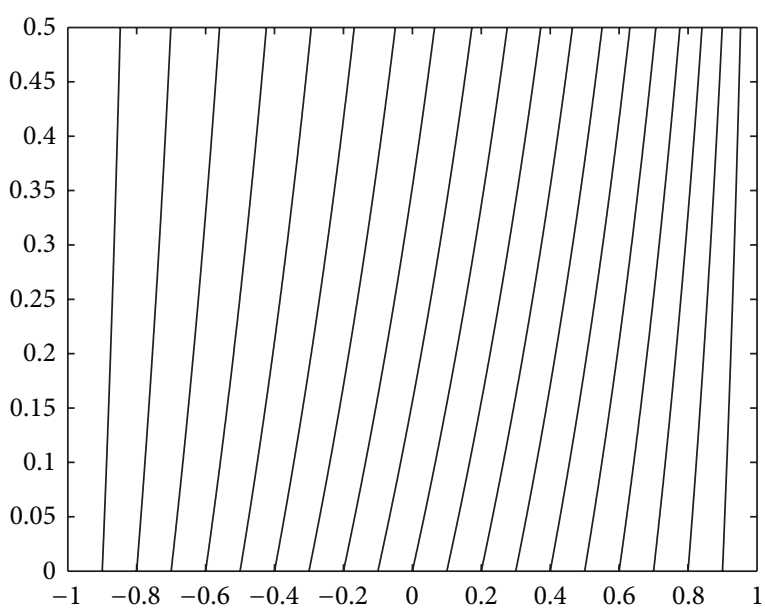

Figure 3: Linear damping $A=1 / 2$.

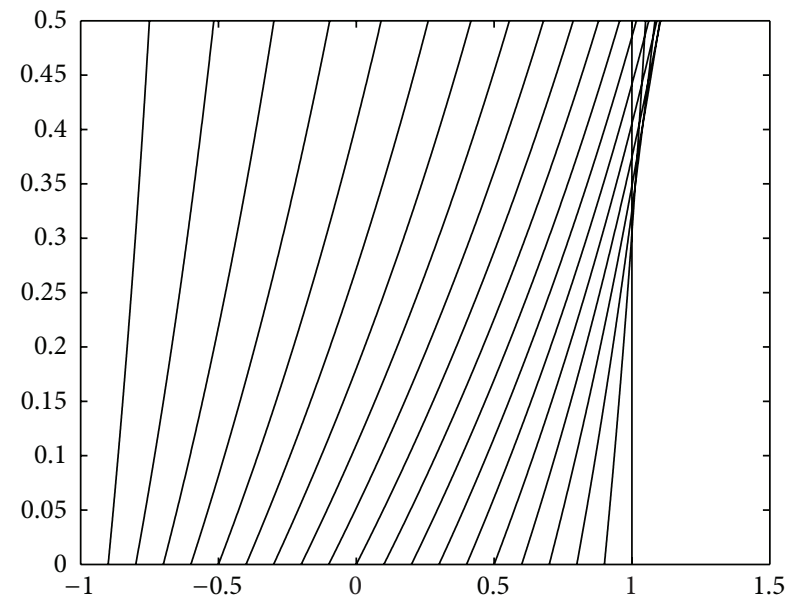

FIgURE 4: Linear damping $A=2$.

Moreover, $r(u, v)$ converges to zero in $L^{p}$ with exponential time decay; that is,

$$
\|r(x, t)\|_{L^{p}(\mathbb{R})} \leq K e^{-M t}\|r(x, 0)\|_{L^{p}(\mathbb{R})} .
$$

\section{Preliminaries}

We start with some preliminaries about the general systems of conservation laws; see [4] chapter 5 . Let $f: \Omega \rightarrow \mathbb{R}^{n}$ be a smooth vector field. Consider Cauchy problem for the system

$$
\begin{gathered}
u_{t}+f(u)_{x}=g(u), \\
u(x, 0)=u_{0}(x) .
\end{gathered}
$$

When $g(u)=0$, the system (12) is called homogeneous system of conservation laws, if $g(u) \neq 0$, the system (12) is called inhomogeneous system or balance system of 
conservation laws. We will work also with the parabolic perturbation to the system (12); namely,

$$
\begin{gathered}
u_{t}+f(u)_{x}=\epsilon u_{x x}+g(u), \\
u(x, 0)=u_{0}(x) .
\end{gathered}
$$

Denote by $A(u)=D f(u)$ the Jacobian matrix of partial derivatives of $f$.

Definition 2. The system (12) is strictly hyperbolic if, for every $u \in \Omega$, the matrix $A(u)$ has $n$ real distinct eigenvalues $\lambda_{1}(u)<$ $\cdots<\lambda_{n}(u)$.

Let $r_{i}(u)$ be the corresponding eigenvector to $\lambda_{i}(u)$. Then, one can see the following.

Definition 3. One says that the $i$ th characteristic field is genuinely nonlinear if

$$
\nabla \lambda_{i}(u) \cdot r_{i}(0) \neq 0 \text {. }
$$

If instead

$$
\nabla \lambda_{i}(u) \cdot r_{i}(0)=0
$$

we say that the $i$ th characteristic field is linearly degenerate.

For the following definitions, see $[5,6]$.

Definition 4. A $k$-Riemann invariant is a smooth function $w_{k}: \mathbb{R}^{n} \rightarrow \mathbb{R}$, such that

$$
\nabla w_{k}(u) \cdot r_{k}(u)=0
$$

Definition 5. A pair of function $\eta, q: \mathbb{R}^{n} \rightarrow \mathbb{R}$ is called a entropy-entropy flux pair if it satisfies

$$
\nabla \eta(u) A(u)=\nabla q(u)
$$

if $\eta(u)$ is a convex function, then the pair $(\eta, q)$ is called convex entropy-entropy flux pair.

Definition 6. A bounded measurable function $u(x, t)$ is an entropy (or admissible) solution for the Cauchy problem (12) if it satisfies the following inequality:

$$
\eta(u)_{t}+q(u)_{x}+\nabla \eta(u) g(u) \leq 0,
$$

in the distributional sense, where $(\eta, q)$ is any convex entropyentropy flux pair.

We consider the general system of Keyfitz-Kranzer type

$$
\begin{aligned}
& u_{t}+(u \phi(u, v))_{x}=0, \\
& v_{t}+(v \phi(u, u))_{x}=0,
\end{aligned}
$$

to get some general observations about this type of system. Let $F(u, v)=(u \phi(u, v), v \phi(u, v))$ in (19), and we have that the eigenvalues and eigenvector of the Jacobian matrix $D f$ are given by

$$
\begin{gathered}
\lambda_{1}(u, v)=\phi(u, v), \quad r_{1}=\left(1,-\frac{\phi_{u}}{\phi_{v}}\right), \\
\lambda_{2}(u, v)=\phi(u, v)+(u, v) \cdot \nabla \phi(u, v), \quad r_{2}=\left(1, \frac{v}{u}\right) .
\end{gathered}
$$

From $(20)$ and (21), we have that $\nabla \phi \cdot r_{1}=0$ and $\nabla Z(u, v) \cdot r_{2}=$ 0 , where $Z(u, v)=u / v$, and then the Riemann invariants are given by

$$
\begin{gathered}
W(u, v)=\phi(r), \\
Z(u, v)=\frac{u}{v} .
\end{gathered}
$$

Lemma 7. The system (1) is always linear degenerate in the first characteristic field. If

$$
(u, v) \nabla \phi(u, v) \neq 0,
$$

then the system (1) is strictly hyperbolic and nonlinear degenerate in the second characteristic field. Moreover,

$$
\nabla \lambda_{2}(u, v) \cdot r_{2}=\frac{2(u, v) \nabla \phi(u, v)+(u, v) H(\phi)(u, v)^{T}}{u}
$$

where $H$ represents the Hessian matrix.

Lemma 8. Let $\eta(u, v) \in \mathbf{C}^{\mathbf{1}}\left(\mathbb{R}_{+}\right)$be a Lipschitz function in a neighborhood of the origin, and let $q(u, v)=\psi(u, v)+$ $\eta(u, v) \phi(u, v)$ be a function, such that $\psi$ satisfies

$$
\nabla \psi(u, v)=((u, v) \cdot \nabla \eta(u, v)-\eta(u, v)) \nabla \phi(u, v) .
$$

Then, the pair

$$
(n(u, v), q(u, v))
$$

is a entropy-entropy flux pair for the system (1). Moreover, if $\eta(u, v)$ is a convex function, then the pair (26) is a convex entropy-entropy flux pair.

\section{Global Existence of Weak Entropy Solutions and Asymptotic Behavior}

We consider the parabolic regularization of the system (1). Namely,

$$
\begin{aligned}
& u_{t}+(u \phi(r))_{x}+a u=\epsilon u_{x x} \\
& v_{t}+(v \phi(r))_{x}+b v=\epsilon v_{x x}
\end{aligned}
$$

with initial data

$$
u^{\epsilon}(x, 0)=u_{0}^{\epsilon} * j_{\epsilon}, \quad v^{\epsilon}(x, 0)=v_{0}^{\epsilon} * j_{\epsilon},
$$


where $j_{\epsilon}$ is a mollifier. In this case, $\phi(u, v)=\phi(r)$, with $r=$ $\sqrt{u^{2}+v^{2}}$. By (20), the eigenvectors and eigenvalues are given by

$$
\begin{gathered}
\lambda_{1}(u, v)=\phi(r), \quad r_{1}=\left(1,-\frac{u}{v}\right), \\
\lambda_{2}(u, v)=\phi(r)+r \phi^{\prime}(r), \quad r_{2}=\left(1, \frac{v}{u}\right) .
\end{gathered}
$$

The following conditions will be necessary in our next discussion:

$$
\begin{aligned}
& \left(C_{1}\right) r \phi(r) \rightarrow 0 \text {, as } r \rightarrow 0, r \phi^{\prime}(r) \neq 0 ; \\
& \left(C_{2}\right) a>b .
\end{aligned}
$$

The condition $\left(C_{1}\right)$ guarantees the strict hyperbolicity to the system (27) according to Lemma 7 , while condition $\left(C_{2}\right)$ ensures the existence of a positive invariant region. Now, we consider the following subset of $\mathbb{R}$ :

$$
\Sigma=\left\{(u, v): \phi(r) \leq C_{0}, 0<C_{1} \leq \frac{u}{v} \leq C_{2}\right\} .
$$

We affirm that $\Sigma$ is an invariant region. Let $h(u, v)=(a u, b v)$, if $(\bar{u}, \bar{v}) \in \gamma_{1}$, where $\gamma_{1}$ is the level curve of $W=\phi(r)$. We have that

$$
(\nabla W \cdot h)(\bar{u}, \bar{v})=(a+b) r \phi^{\prime}(r)>0,
$$

and if $\bar{u}, \bar{v} \in \gamma_{2}$, where $\gamma_{2}$ is the level curve of $Z=u / v$, we have that

$$
(\nabla Z \cdot h)(\bar{u}, \bar{v})=(a-b)\left(\frac{u}{v}\right)>0 .
$$

By Theorem 14.7 of [5], $\Sigma$ is an invariant region for the system (27). It is easy to verify that $(a u, b v)$ satisfies the condition $H_{1} \cdots H_{5}$ in [3]; thus, we have the following Lemma.

Proposition 9. If $\left(u_{0}, v_{0}\right) \in \Sigma$ and the $C$ condition holds, then the Cauchy problems (27), (28) have a global weak entropy solution.

Now, for the global behavior of solutions, using ideas of the author in [7], we construct the following entropy-entropy flux pairs as follows:

$$
n(r)=r^{m}, \quad m \geq 2 .
$$

From (25), we have

$$
q(r)=(m-1) \int_{0}^{r} s^{m} \phi^{\prime}(s) d s+r^{m} \phi(r) .
$$

Integrating by parts, we have that

$$
q(r)=m \phi(r)-m(m-1) \int_{0}^{r} s^{m-1} \phi(s) d s .
$$

Let $M=\sup _{(u, v) \in\left[0,\|u\|_{L^{\infty}}\right] \times\left[0,\|v\|_{L^{\infty}}\right]}\{\phi(r)\}$. Then, we have that

$$
|q(r)| \leq 2 m M r^{m} .
$$

Multiplying (1) by $\nabla \eta$, we have that

$$
\eta(r)_{t}+q(r)_{x} \leq-3 m M r^{m} .
$$

Now, we choose $h(x) \in C^{2}(\mathbb{R})$ as a function, such as $\left|h^{\prime}(x)\right| \leq$ $1,\left|h^{\prime \prime}(x)\right| \leq 1$, and $h(x)=|x|$ for $|x| \geq 1$ and set $k(x)=$ $e^{-h(x)}$. Then, $k^{\prime}(x) \leq k(x)$. Multiplying by $k(x)$ in (37) and integrating over $x$, we have

$$
\frac{d}{d t} \int_{-\infty}^{\infty} \eta(r) g(x) \leq \int_{-\infty}^{\infty} q(r) k^{\prime}(x)+-3 m M \int_{-\infty}^{\infty} r^{m} d x .
$$

By the inequality (36), we have

$$
\frac{d}{d t} \int_{-\infty}^{\infty} \eta(r) k(x) d x \leq-m M \int_{-\infty}^{\infty} r^{m} k(x) d x .
$$

If $\psi(t)=\int_{-\infty}^{\infty} \eta(r) k(x) d x$, we have

$$
\frac{d}{d t} \psi(t) \leq-m M \psi(t) .
$$

By Gronwall's inequality, we have

$$
\psi(t) \leq e^{-m M t} \psi(0) .
$$

Thus, we have

$$
\left(\int_{-\infty}^{\infty} r^{m}(t) k(x) d x\right)^{1 / m} \leq e^{-M t}\left(\int_{-\infty}^{\infty} r^{m}(0) k(x) d x\right)^{1 / m} .
$$

Passing to limit $m \rightarrow \infty$, in (42), we have the inequality (10).

\section{Conflict of Interests}

The authors declare that there is no conflict of interests regarding the publication of this paper.

\section{Acknowledgments}

The authors would like to thank Professor Laurent Gosse for his suggestions and review, Professor Juan Galvis for his many valuable observations, Professor Yun-Guang Lu for his suggestion for this problem, and the reviewer for his many valuable suggestions.

\section{References}

[1] B. L. Keyfitz and H. C. Kranzer, "A system of non-strictly hyperbolic conservation laws arising in elasticity theory," Archive for Rational Mechanics and Analysis, vol. 72, no. 3, pp. 219-241, 1980.

[2] N. D. Cristescu, Dynamic Plasticity, World Scientific, River Edge, NJ, USA, 2007.

[3] G.-Q. Song, "Existence of global weak solutions to a symmetrically hyperbolic system with a source," Revista Colombiana de Matemáticas, vol. 42, no. 2, pp. 221-232, 2008. 
[4] A. Bressan, Hyperbolic Systems of Conservation Laws: The OneDimensional Cauchy Problem, Oxford University Press, Oxford, UK, 2000.

[5] J. Smoller, Shock Waves and Reaction-Diffusion Equations, Springer, New York, NY, USA, 1994.

[6] H.-m. Yu, "Large time behavior of entropy solutions to some hyperbolic system with dissipative structure," Acta Mathematicae Applicatae Sinica, vol. 29, no. 3, pp. 509-516, 2013.

[7] E. Y. Panov, "On the theory of entropy solutions of the Cauchy problem for a class of non-strictly hyperbolic systems of conservation laws," Sbornik: Mathematics, vol. 191, no. 1, pp. 127$157,2000$. 


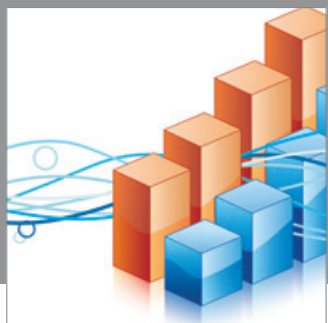

Advances in

Operations Research

mansans

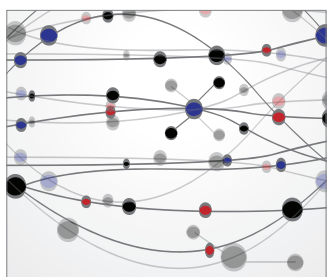

The Scientific World Journal
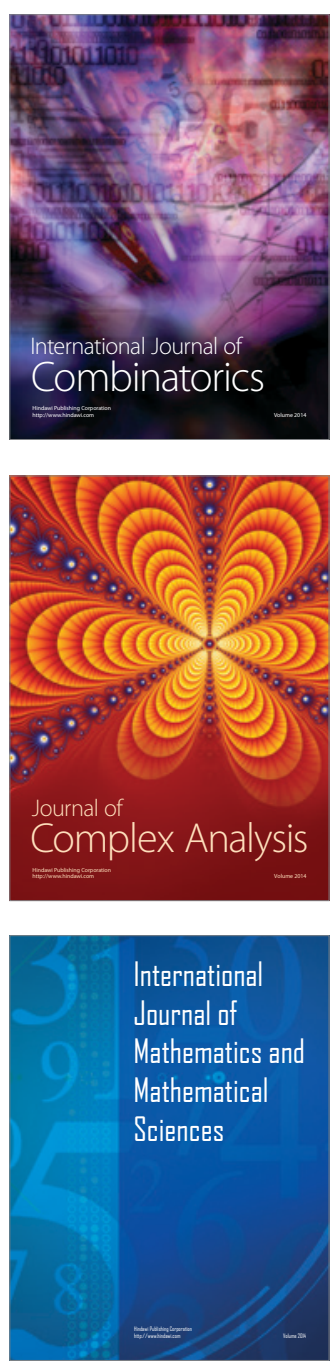
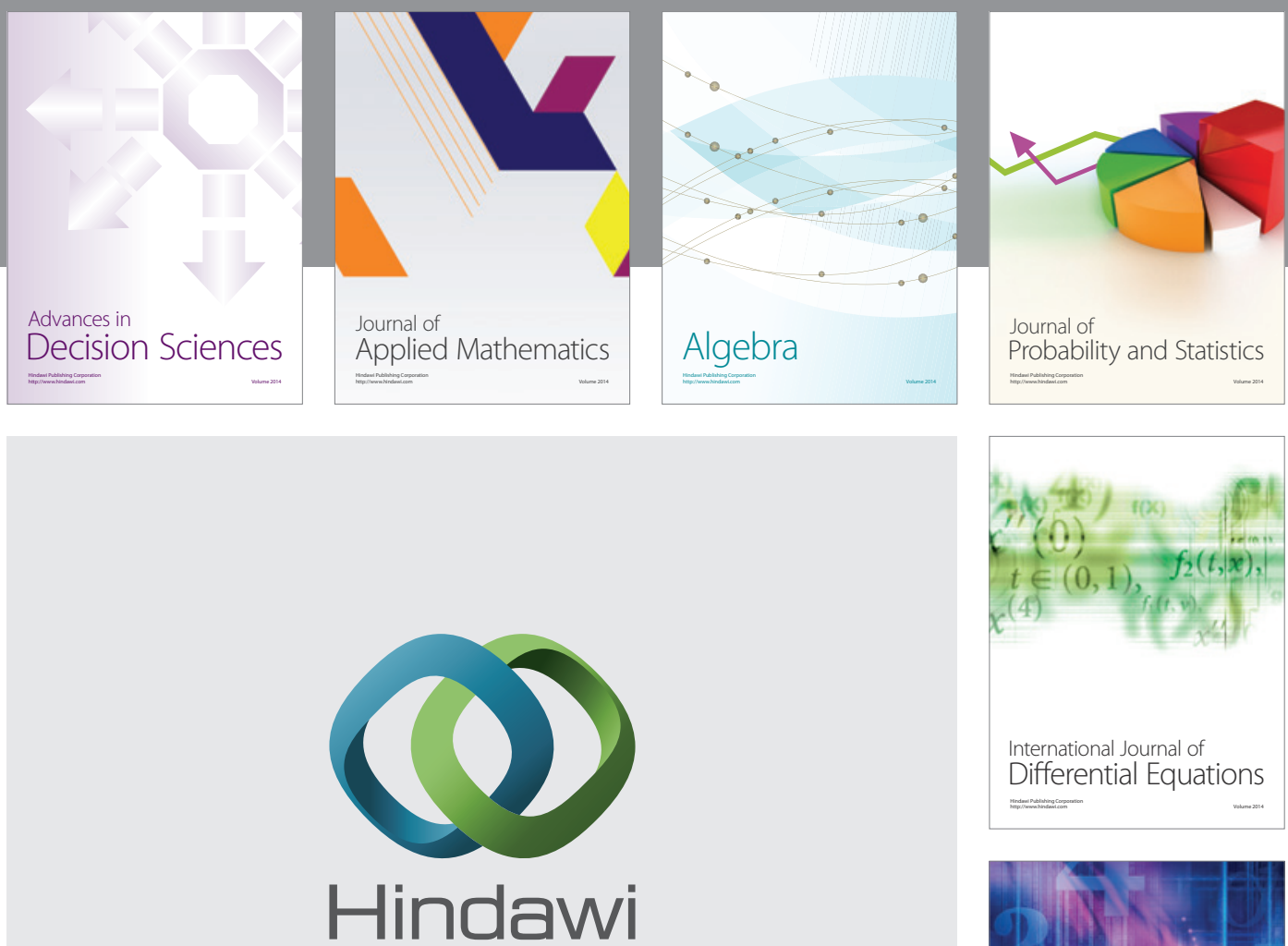

Submit your manuscripts at http://www.hindawi.com
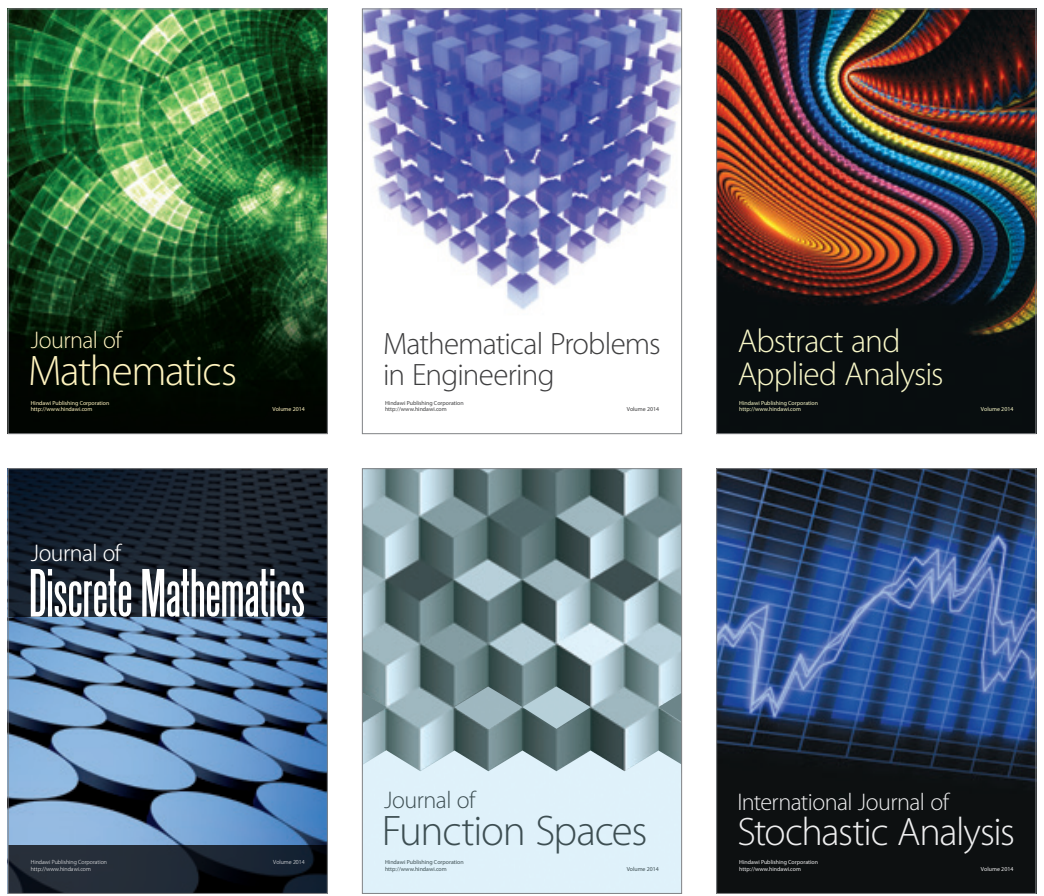

Journal of

Function Spaces

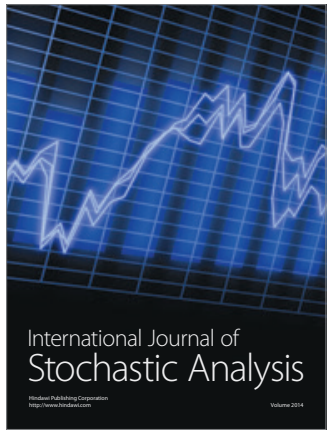

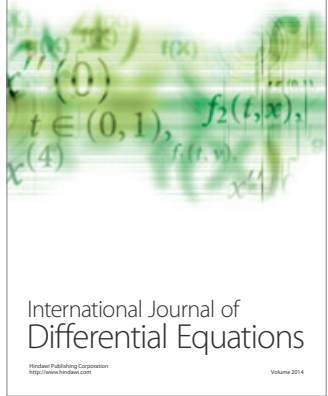
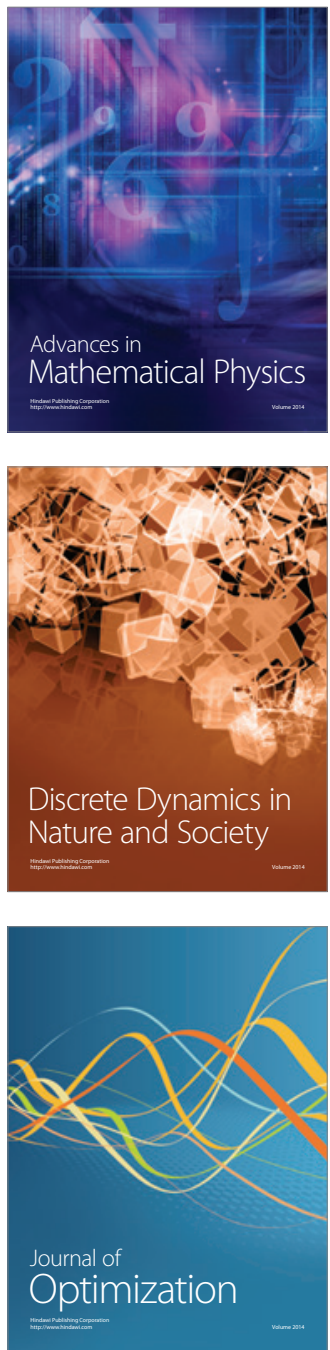